\title{
Designing the primary mirror support for the E-ELT
}

\author{
Jan Nijenhuis, Jeroen Heijmans, Remco den Breeje, René Hazelebach, Jan de Vreugd,Will \\ Crowcombe, Daniel Naron, Erik Fritz, Guiseppe Borghi, TNO Technical Science, Stieltjesweg 1, \\ 2623 CK Delft, The Netherlands, Ramon Navarro, Astron, Dwingeloo, The Netherland, \\ Luca Sillari, Enrico Sambenedetto, Josef Eder, Airworks, Italy \\ Fred Kamphues, TMT, USA
}

\begin{abstract}
Described is the M1 segment support, as designed by TNO in the period 2015- 2016. The design has significantly changed and improved compared to the earlier designs. During the period 2009-2010 prototypes for the primary mirror support of the E-ELT have been developed. These have been extensively tested by ESO. Design improvement were found to be necessary, especially in the field of manufacturability and maintainability. Furthermore, the technical performance had to improve in specific areas as well. This has evolved into a new specifications which have resulted in a new design for the segment support structure. The design rules that have led to the prototype design have been maintained but the implementation has been much improved. Also considerable improvement has been obtained with respect to the dynamic behavior. Accessibility and visibility on all parts and subsystems has changed such that everything is now clearly visible. Despite the increased performance no mass increase has been recorded meaning that more efficient use has been made of the material.

The active means to influence the segment shape by use of the warping harness has been completely redesigned. A very important quality that has been achieved is simplicity. Hence a minimum amount of components is used. Reliability and safety are other aspects that have been greatly improved compared to the prototypes. The design for the M1 segment support provides an answer that not only performs to specification but one that can be operated in a telescope environment, all 798 of them.
\end{abstract}

Keywords: Opto-mechanics, Static-determined, warping harness, E-ELT, M1 support structure, design rules, mirror segment.

\section{INTRODUCTION}

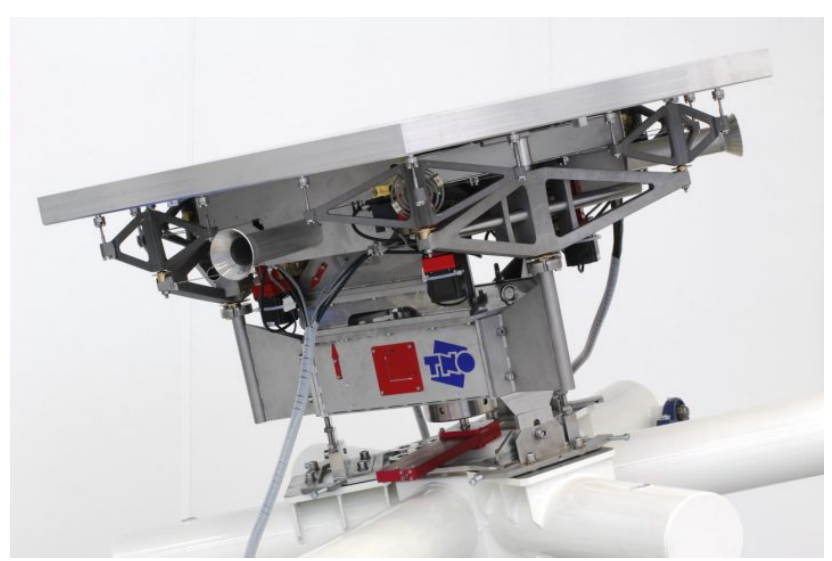

Figure 1: The 2010 prototype for the M1 segment support structure.
In 2010 TNO delivered three prototypes for the M1 segment support structure to ESO. These prototypes were meant to be tested extensively in a specific test setup. The results of this evaluation have been published by ESO in ref 1 . It was concluded that although the prototypes worked well that there was a need for improvement. Performance, maintenance and cost were the main drivers.

In February 2015 the contract was signed to design and built an engineering model and four qualification models for the M1 segment support structures between ESO and the Dutch consortium VDL/TNO/NOVA. TNO is responsible for the design and testing assisted by NOVA while VDL-ETG is responsible as the prime contractor for the total

development, manufacturability and the possible future series production. The Italian company Airworks together with Mr. Eder have been responsible for the majority of the mechanical analyses. 
As a result of the changed technical requirement specification and the evaluation of the prototype it was decided not to just upgrade the design but push for a new design based on the proven principles of the prototype but with much improved maintenance characteristics. Furthermore bringing down the production cost was felt as an urgent need. This has led to a new design that is very promising in meeting these goals. At present VDL-ETG is building the engineering model which should be finished around the end of July 2016. This model will be tested extensively. To make sure that these tests will be successful much effort was put in the preparation of these tests. The main critical tests concern the measurement of the eigen frequencies and to measure the loads at the support points of the segment in the operational configurations.

\section{DESIGN DESCRIPTION}

Before going into the details and explaining the design updates and improvements a short summary will be given about the main components, where they are located and what their main function is. It all is illustrated in Figure 2.

The M1 mirror is split into 798 hexagonal shaped segments. At the back each segment is supported by the same support structure consisting of three whiffletrees. In the lateral direction each segment is supported by an identical membrane. Both support structures are connected to the Moving Frame (MF) which acts as an intermediate body before being connected to the telescope structure itself. With the addition of a clocking strut between MF and segment a static determined connection between segment and MF is realized. Together this is called the Segment Assembly (SA). Three actuators called PACT (Position ACTuator) are mounting in between the telescope structure and the MF to control the segment in piston (translation along optical axis of segment) and tip/tilt motion (rotation about two perpendicular axes which are oriented parallel to the segment surface). This is done to compensate for gravity deformations of the telescope structure. Finally three flexures (Moving frame flexure) connected to the Fixed Frame (FF) make sure that the MF can only move in piston and tip/tilt i.e. lateral motions and rotations around the optical axis are prevented. The assembly providing the connection between SA and the telescope structure is called subcell structure.

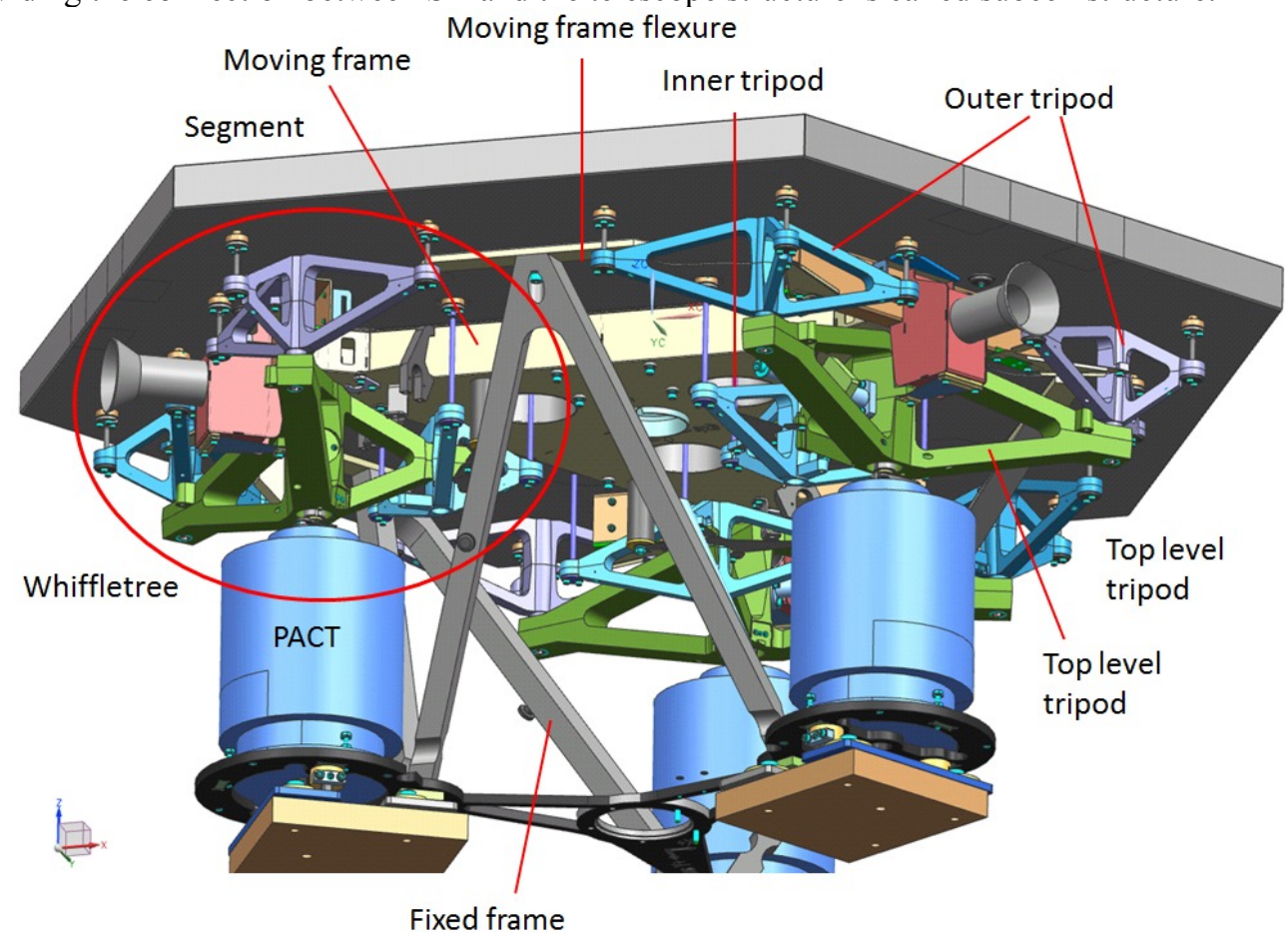

Figure 2: The definition of the various sub-assemblies of the segment support structure.

\section{MAIN REQUIREMENT CHANGES}

Although many details of the technical requirement specification were improved/changed three main items have changed in such way that they had major impact on the design of the M1 segment support structure. These are: 
1. The required natural frequencies have increased. These frequencies will have to be verified by testing while analysis was considered sufficient for the prototypes. That means that some margin now has to be taken for the obtained frequency values because improving the natural frequencies after testing is a rather hopeless mission when fundamental errors are discovered. Altogether this meant that the stiffness requirement for the axial and lateral support of the segment increased by $20 \%$.

2. To realize the best possible stiffness for the segment axial support it was decided to require that the PACTs were no longer to be mounted to the side of the Fixe Frame. Instead the PACTs are to be supported directly from underneath thereby creating the shortest load path from segment to the telescope structure.

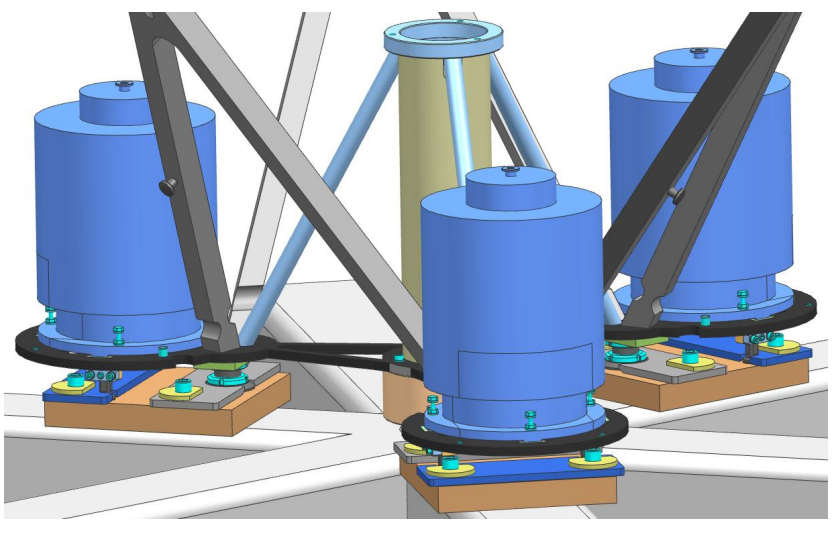

Figure 3: Axial mounting of the PACTs (new design).

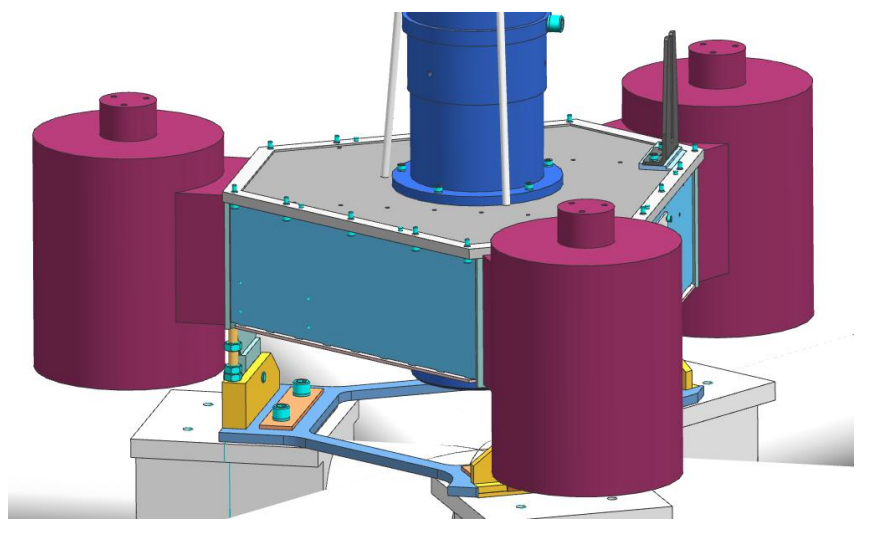

Figure 4: Lateral mounting of the PACTs (prototypes).

3. Originally each Fixed Frame was equipped with an extractor to allow the removal and reinstallation of the segment assembly. This extractor is basically an actuator that allows and removal of the segment for maintenance purposes. However it was found that this was a real cost driver to have this installed in every of the 798 segments. Therefore it is now required to provide one extractor. This will act as tool that can be inserted in every Fixed Frame. To make this possible a hole is provided in the telescope structure that allows inserting the extractor from underneath into the Fixed Frame.

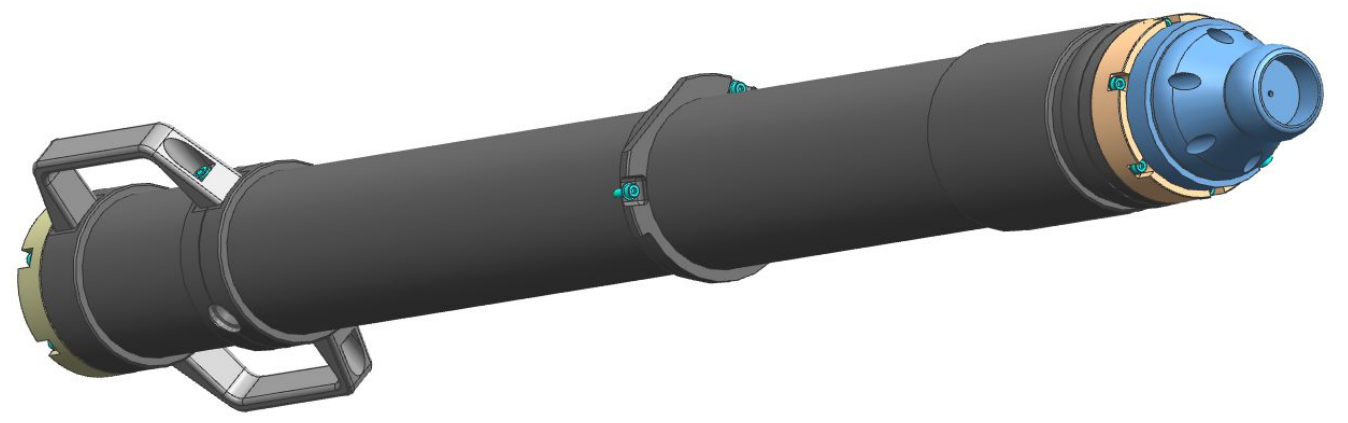

Figure 5: A separate extractor is now required and has become a maintenance tool.

\section{MAINTAINABILITY}

Not just the requirements have changed. Also the emphasis on maintainability was enhanced. From a functional and performance point of view the prototype was very capable. However when coming to accessibility and maintainability it performs insufficiently. It was obvious that an upgrade of the design was necessary to improve these features while maintaining or improving its function and performance. The key feature in achieving these goals is formed by the a specific sub-assembly called Moving Frame (MF). This sub-assembly has a strong integration function but its main two function are:.

1. It is the intermediate body between the telescope and the segment and it minimizes the impact of the PACT operation on the Surface Form Error (SFE). 
2. It is one of the most important elements in the stiffness chain from segment to telescope structure that determines the natural frequencies for Tx, Ty (lateral modes) and Rz (clocking).

While Figure 2 gives a general overview of the new design Figure 6 and Figure 7 show the design of the Moving frame for the present design and the prototype. Figure 7 clearly shows that the volume of the Moving Frame used to be big. Now it has become much smaller making accessibility and therefore maintainability much better. The basis of the new MF is a triangular box which corners coincide with the support points for the PACTs. The height of the box is now just over $60 \mathrm{~mm}$ while the original box was more than double that height. Despite the big volume decrease its stiffness has improved. This is because of the more efficient use of the available material and by minimizing the load path length between segment and telescope structure. The box is made of $1.5 \mathrm{~mm}$ thick, stainless steel plates . The plates are welded together using laser welding. This provides a continuous connection between the sheet metal parts while the laser guarantees that power transmission to the box is minimized. That results in a box that does not warp due to differential thermal expansions during the welding process.

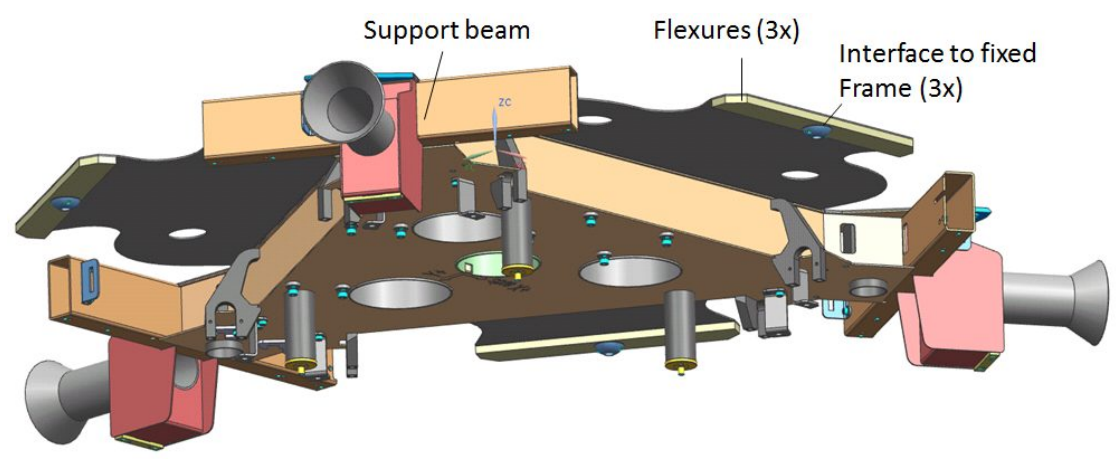

Figure 6: The new design for the moving frame.

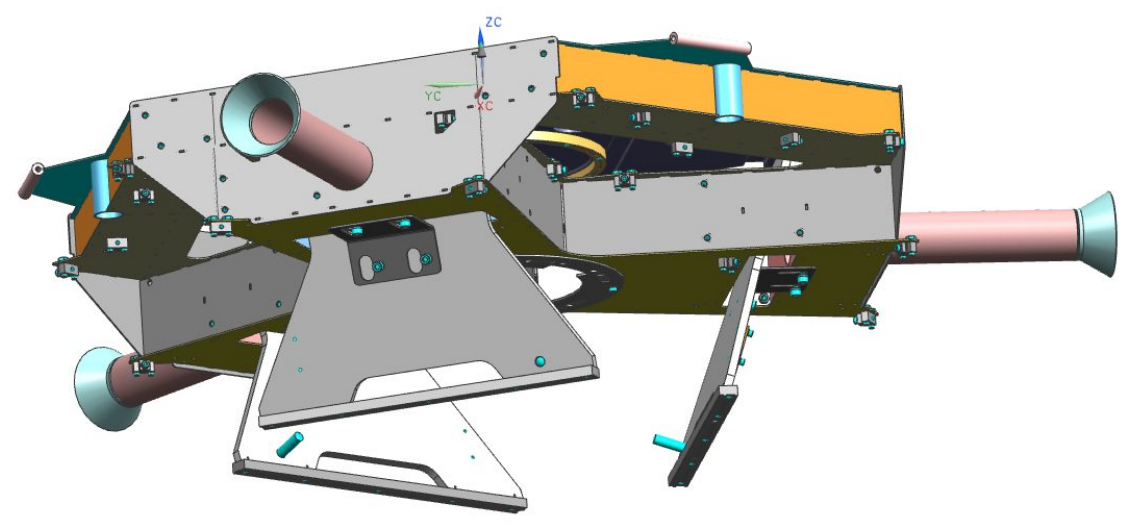

Figure 7: Prototype design for the moving frame.

The new MF had one serious consequence for the WT. Originally the WT configuration consisted of a top level tripod supporting three more tripods located at a physically higher level. This made separate lateral supports for these tripod necessary. This configuration is still possible for the six outer tripods but the three inner ones would interfere with the MF. For that reason the inner tripods are now located at the same level as the Top Level Tripods and their lateral supports are now combined (Figure 2). This configuration for the WT is unique. The usual configuration for support systems making use of whiffletrees have all tripods at the same level while the TNO prototype had all inner and outer tripods at a physically higher level. Now a mixture of the two configurations is used.

Figure 9 shows the subcell structure as it used to be for the prototype. Figure 8 shows the new design and the difference is very obvious. By using a truss structure for the Fixed Frame a very open structure is created providing easy access to equipment inside the subcell or hanging down from the MF. Originally the PACTs were hanging laterally on the FF 
(Figure 4) thereby more or less closing the gaps between the vertical plates shown in Figure 9. This made accessibility bad. The requirement change to support the three PACTs in the axial direction made the conversion to six struts for the FF possible.

The FF is attached to the telescope structure at three points. The FF is also attached to the three horizontal sections of the MF-flexures. The most important benefits of this solution are:

1. The accessibility is greatly improved because the obstruction by the FF struts is minimized.

2. The struts are loaded in tension/compression which means that optimal use of material is made to get the highest stiffness for the connection between MF and FF.

3. The length of the struts is maximized which means that the angular deflection of the struts is minimized (compensation of the flexure shortening). Hence the cross section of the elastic hinge at the lower end of the struts is maximized thereby further improving the tension/compression stiffness of the struts.

4. Visibility of the extractor operation has become much better. When inserting the extractor from below into the central tube of the FF one can see the top end of the extractor very well especially during the engagement of the extractor with the MF. For such delicate operation it is seen as a great advantage when the operator has full visibility on this operation. Now the operator can also witness that the connection between extractor and MF is right. This reduces the risk for the extraction of the SA.

5. The three lower connection of the six struts are interconnected by a triangular plate that also supports the central tube that accommodates the extractor. The top end of that tube is connected by three struts to the lower support points of the ff. Hence a very efficient combination of functions has been realized. The triangular plate has three circular extensions that serve as temporary supports for the PACTs. The thickness of the plates is sufficient to make this possible. However its stiffness properties are not fit for purpose. For that reason separate supports underneath these mounting platforms are provided to realize the required stiffness between the PACTs and the telescope structure.

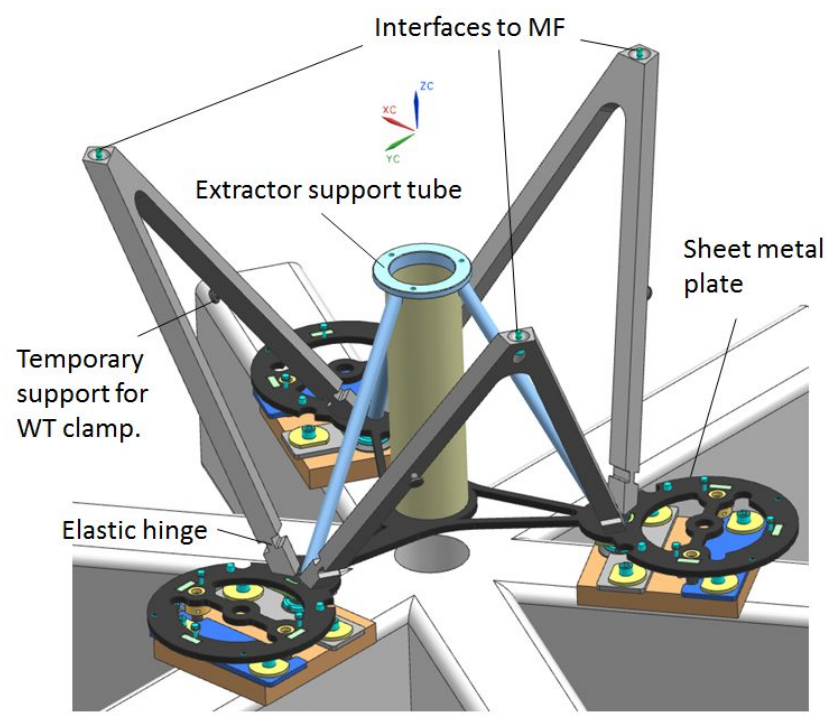

Figure 8: The new design for the Fixed Frame.

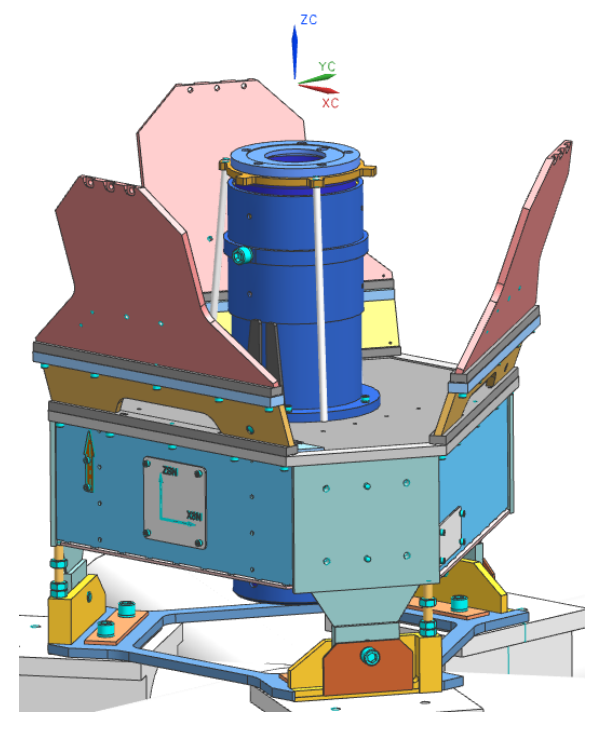

Figure 9: The prototype Fixed Frame with extractor.

Warping Harnesses (WH) are used to slightly deform the segment thereby compensating for some residual SFE. The WH are designed for minimum maintenance need. However due to the large number of WH $(>7000)$ it is likely that an actuator has to be replaced either due to failure or due to a preventive maintenance action. Accessibility to the WH is therefore very important. To further ease the removal and installation of a $\mathrm{WH}$ a quick release feature has been implemented that is operated by hand. After disconnecting the electrical cable connector the actuator can be removed by releasing two bolts. These bolts cannot fall out. This operation applies to both type of actuators (Figure 10). For each Top Level Tripod (Figure 11) two WHs are provided. Three of the six outer tripods are also equipped with WHs.. 
Further details about the WH are provided in the next chapter.
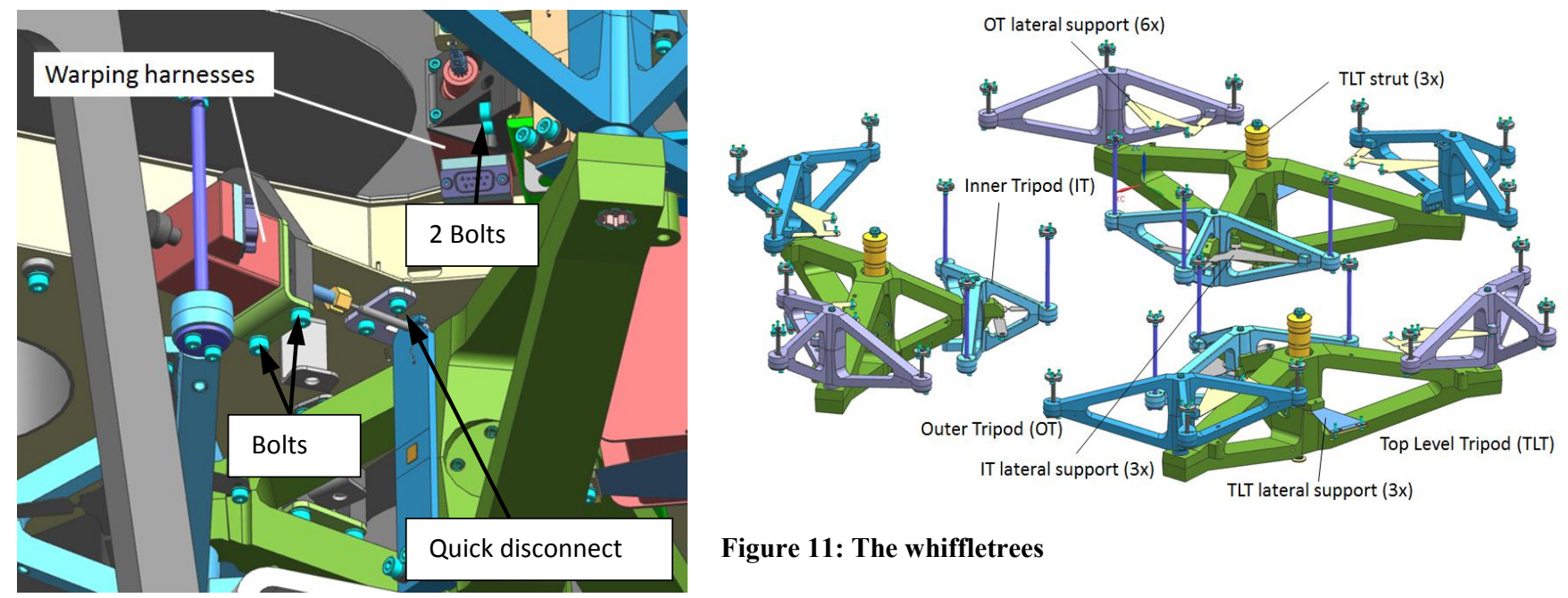

Figure 11: The whiffletrees

Figure 10: Warping Harness installation in the Segment Assembly.

All 798 Fixed Frames will have to be aligned with respect to the telescope structure. It is anticipated that iterations are needed to do so. That means that alignment must be straight forward and with the need for minimal iterations. To do this three push-pull screws are attached to the PACT support platforms. Opposite adjustment of the two parallel screws rotates the FF while identical adjusting results in a translation. Adjusting the other screw (perpendicular to the two parallel ones) provides the third DOF adjustment. Locking of the FF to the telescope structure is done using three clamping units located where the three pairs of FF struts meet.

Crucial for a successful alignment operation are stiffness and friction because these two parameters determine the magnitude of the alignment stick-slip effect which is the minimal step size. A high stiffness and low friction minimizes the step size for adjustment. The stiffness of the alignment tooling and the FF has been optimized. Most parts that are mechanically loaded during alignment are loaded in tension/compression which guarantees highest stiffness. Some parts however will be loaded in bending which reduces stiffness considerably. This is compensated by making these parts very solid.

Play in the push-pull connection is not very important because it does not influence the alignment resolution. 


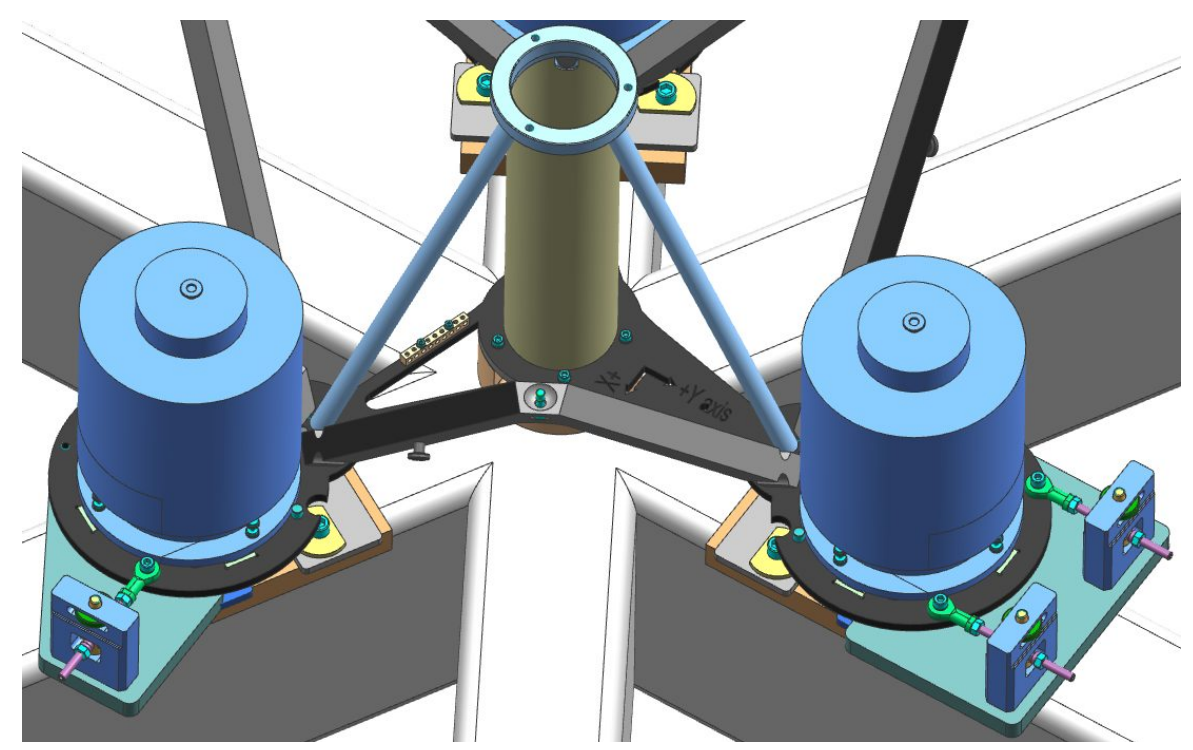

Figure 12: Alignment of the FF by use of three push-pull screws.

\section{COST REDUCTION FOR SERIES PRODUCTION}

Having made the prototypes provides a lot of valuable data to estimate the unit price for series production. It also highlights where possible savings with highest result are to be obtained. These savings are linked to:

- Reduction of labor cost.

- Avoid high accuracy parts.

- Simplifying mechanical parts.

- Vacuum compatibility.

- Selection of the most cost effective production processes.

Some examples that demonstrate these improvement are given below.

Reduction of labor cost is especially obtained by eliminating the need for alignment of the SA and the FF during assembly. An extensive tolerance analysis was performed to prove this. This analysis also proved that readily available machining accuracies is sufficient to meet this goal. Testing the engineering model will further prove the validity of this approach. In addition this reduces the ESO maintenance effort in case parts have to be replaced.

The MF and FF have much reduced in complexity as has been shown in the previous chapter. E.g. the FF struts are obtained by water jet cutting of $30 \mathrm{~mm}$ thick steel plate with minimal material loss. Many strut pairs (V-shaped) can be made from one steel plate because one strut pairs fits in another strut pair. It also nearly halves the time to produce one strut pair.

The MF is more simple and smaller than it used to be. Hence on top of performance and accessibility improvement it has also become cheaper.

Figure 14 shows the design of the WH for the prototype. It consists of a bracket, worm-gear transmission, steppermotor, clock spring and coupler. All of them are either too expensive or not really needed. Much experience was obtained already by the TMT-team to produce a cost effective WH. This knowledge has been shared with TNO/VDL. This has resulted in a complete redesign of the WH. They are now very similar to each other although typicalc differences exist because of the specific requirement that have to be met. Both units make use of the same stepper motors apart from the electrical connector that is required by ESO.

The clock spring has been replaced by a leaf spring that is bolted to the tripods. The combination of actuator step size and leaf spring eliminated the need for a separate gearbox. The self-locking quality of the actuator made the worm gear superfluous. Finally the need for the flexure based coupling between tripod and MF was eliminated because of the new requirement for the WHs to provide a "dead band". This band is sufficiently big that it can accommodate the mechanical 
tolerances that determine the relative position of actuator and leaf spring. Having implemented all these changes it will be obvious that the price of the WH has decreased.

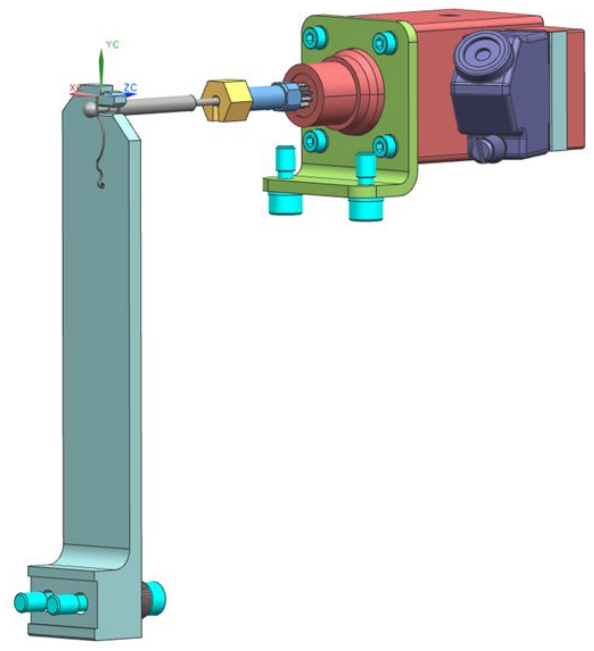

Figure 13: The Warping Harness.

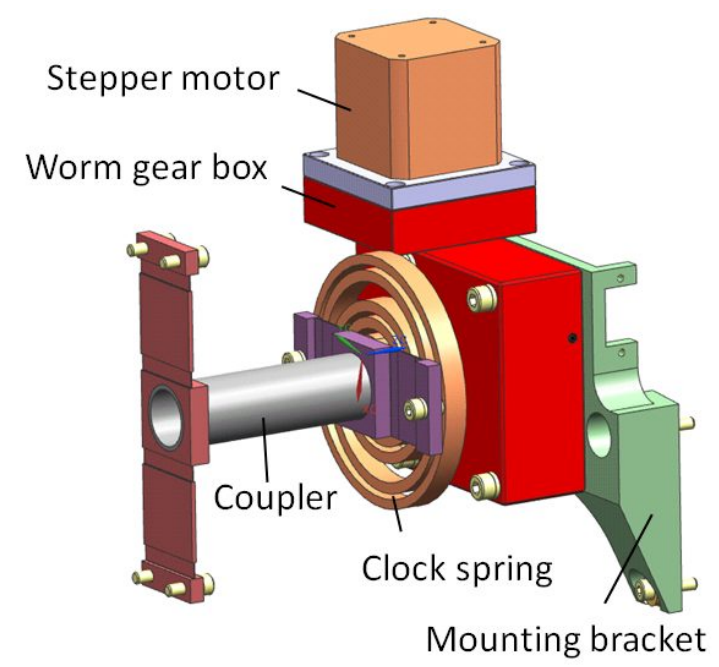

Figure 14: The prototype Warping Harness.

The need for wire erosion cutting has been minimized. Being a rather slow and therefore expensive process its application has been limited to those parts where other production processes are less cost effective. Typically this concerns some of the elastic hinges used in the struts of the axial support.

Machining parts in one operation without the need to e.g. put it upside down to finish machining is another way to realize a cost reduction. This applies e.g. to the tripods. Original the tripods had to be machined from top and bottom.

Removing the 798 extractors and replacing them by one extractor tool is a big cost saving. However it means that more handling is to be done before an SA can be removed from the telescope. This is avoided by making the design such that the extractor can remain inside the subcell because it does not interfere with normal operation of the telescope.

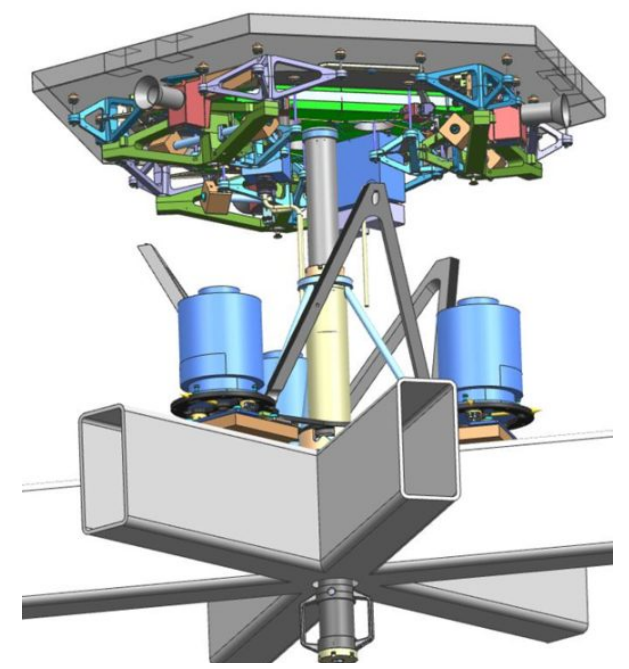

Figure 15: The extraction of the Segment Assembly.
Not everything has become cheaper. Some of the off the shelve parts have become much more expensive since the prototypes were manufactured. Finding more cost effective alternatives is still subject of discussion.

Finally vacuum compatibility has proven to be the biggest cost driver. The requirement to put the SA into the ion beam figuring facility and/or the coating chamber without the need to remove actuators, connectors etc. proved to be very difficult in relation to the involved cost. Making actuators vacuum compatible is something different that modifying then so they survive the vacuum. This has everything to do with the outgassing products in vacuum which will contaminate the equipment or influencing the processes that have to be done in vacuum. Furthermore the time it takes to realize certain vacuum level is also an important parameter in the manufacturing process of the segments. Water plays an important role in this because much water is absorbed in the standard materials that are used for mechanical equipment. Pumping down the vacuum chamber causes that the water evaporates from these material. Unfortunately this is a time consuming process. Usually this is expedited by heating up the unit provided the unit allows this. 
For the electrical connectors similar considerations apply. Connectors are cheaper than actuators but their number is bigger For space applications these problems have been solved at the expense of very expensive parts. For the E-ELT this solution is too expensive even taking into account the high number of actuators $(>7000)$ that is needed. This meant that a new compromise had to be found concerning the vacuum requirements and the cost involved. In general this meant that a compromise was found in accepting less stringent vacuum requirements and to use non-standard components which are still affordable.

\section{PERFORMANCE PREDICTION.}

So far little has been said about the performance of the system. The most important ones concern the Surface Form error and the dynamic performance. The requirement of $25 \mathrm{~nm}$ rms for the SFE has been met. Most of this is caused by the SFE that remains after mass balancing. The SFE induced by mechanical tolerances, temperature changes, thermal gradients, PACT piston movement etc. amount approximately $13 \mathrm{~nm}$ rms. Especially the thermal performance is very good. For a temperature change of $20^{\circ}$ only $2.3 \mathrm{~nm}$ rms SFE was predicted. This is even better than what was obtained for the prototypes. Furthermore the SFE due to mechanical tolerances on the segment support structure amounts less than $5 \mathrm{~nm}$ rms. Similarly the contributions of the segment shape tolerances causes around $10 \mathrm{~nm} \mathrm{rms}$.

Due to the change concerning the dynamic performance much has been done to improve the natural frequencies. TNO has been successful in this by meeting the requirements with a general margin of $10 \%$ by design. Usually making a structure more stiff also means that its mass will go up. Here this does not apply. Mass has hardly changed which means that the design has become more efficient.

\section{CONCLUSIONS}

1. The design of the M1 segment support structure and the WH has much improved since the delivery of the prototypes to ESO in 2010.

2. Accessibility and maintainability of the SA has greatly improved by minimizing the size of the MF and reducing the FF to six bars.

3. Many cost savings have been implemented although the result of it is threatened by the cost increase of off-theshelve components and the vacuum compatibility requirements.

4. All major performance requirements are met.

5. The thermal sensitivity of the systems is remarkably small.

6. Assembly of the SA and FF will be quick because there will be no need to spend valuable time for alignment.

\section{FUTURE ACTIVITIES}

The design work is done but manufacturing and testing is still coming. Although much bread boarding has been done there might be surprises so an interesting period is ahead of us that will prove to be interesting to report about in the future. The two main tests to be performed are:

1. Measurement of the loads applied by the segment to the axial support. Being static determined these loads can be predicted easily but will it be like that in reality? A special tool has been designed to measure the strut loads. Much work was done to find proper load cells that fit inside a dummy mirror and which are only sensitive to the tension/compression loads of the struts on not to spurious loads like gravity effects on the load cells. The tool that was designed (Figure 16) has recently be delivered to TNO/VDL and first results are very promising.

2. Measuring the eigen frequencies because this will have great impact on the control of the E-ELT. 


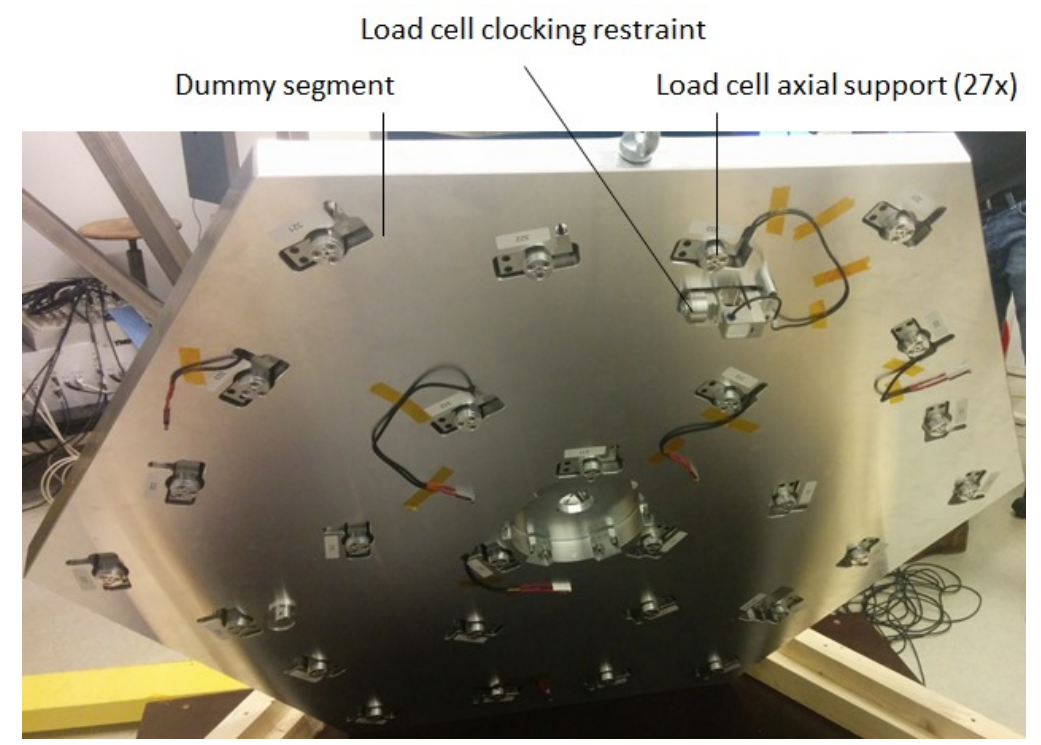

Figure 16: Dummy mirror with load cells.

Other interesting projects are coming for the E-ELT like:

- The support structures for the M2 and M3 mirrors. These will be similar to the support structure for the segment however much bigger because of the sizes of M2 and M3 (Ø4.25m and Ø4m).

- Over 2400 PACTs are needed. They have to be very accurate (nanometer level) and provide excellent dynamic behavior..

- The segment have to be phased relative to each other. Not just when integrating the E-ELT but each time a segment has been removed for coating and is reinstalled. This has to be done with nanometer accuracy and in a short timescale. Hence this will be very challenging.

\section{ACKNOWLEDGEMENT}

The work reported in this paper was conducted at TNO/VDL under a contract with ESO in Garching (Germany). We acknowledge ESO for granting TNO/VDL the contract for the development of such magnificent instruments as the support structure for the primary mirror segment of the E-ELT.

Also TMT is acknowledged for the discussions and information they have provided and which has helped to make such a beautiful and performing instrument as described in this paper.

\section{REFERENCES:}

REF. 1: Jan Nijenhuis, Ben Braam, TNO Technical Science. Roger Hamelinck, Entechna. The opto-mechanical performance prediction of thin mirror segments for E-ELT.. SPIE conference Astronomical telescopes and instrumentation, Amsterdam 2012.

REF. 2: Jan Nijenhuis, Roger Hamelinck, Ben Braam, Marc Cayrel. Meeting highest performance requirements for lowest price and mass for the M1 segment support unit for E-ELT. SPIE conference San Diego 2010.

REF. 3: Jan Nijenhuis, Roger Hamelinck. The optimization of the opto-mechanical performance of the mirror segments for the EELT. SPIE, Integrated modelling, Kiruna 2011. 